\title{
Novel lattice models for porous media
}

Link to publication record in Manchester Research Explorer

\section{Citation for published version (APA):}

Jivkov, A. P., \& Olele, J. E. (2011). Novel lattice models for porous media. In Procceding of the MRS XXXV International Symposium Materials Research Society.

\section{Published in:}

Procceding of the MRS XXXV International Symposium

\section{Citing this paper}

Please note that where the full-text provided on Manchester Research Explorer is the Author Accepted Manuscript or Proof version this may differ from the final Published version. If citing, it is advised that you check and use the publisher's definitive version.

\section{General rights}

Copyright and moral rights for the publications made accessible in the Research Explorer are retained by the authors and/or other copyright owners and it is a condition of accessing publications that users recognise and abide by the legal requirements associated with these rights.

\section{Takedown policy}

If you believe that this document breaches copyright please refer to the University of Manchester's Takedown Procedures [http://man.ac.uk/04Y6Bo] or contact uml.scholarlycommunications@manchester.ac.uk providing relevant details, so we can investigate your claim.

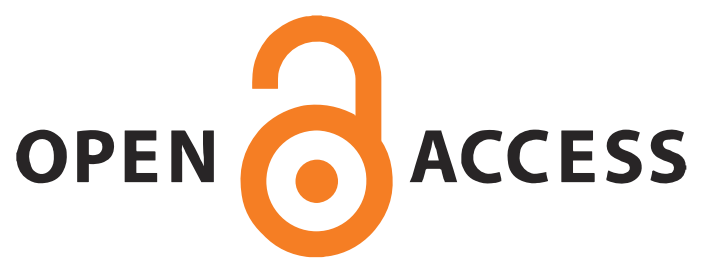




\title{
Novel Lattice Models for Porous Media
}

\author{
Andrey P. Jivkov ${ }^{1,2}$ and Joseph E. Olele ${ }^{2}$ \\ ${ }^{1}$ RCRD, University of Manchester, Williamson Building, Manchester, M13 9PL, UK. \\ ${ }^{2}$ MACE, University of Manchester, George Begg Building, Manchester, M13 9PL, UK.
}

\begin{abstract}
Network models of porous media are beneficial for predicting evolution of macroscopic permeability. This work proposes novel models based on truncated octahedral support. Systems with different pore coordination spectra for a given average coordination number can be constructed to match experimental data. This feature, and the allowed pore coordination of 14 , make the proposed models more realistic and flexible than existing models with cubic support. Experimental data for two sandstones with substantially different properties are used to demonstrate the models' ability to predict permeability. A strategy for calculating its evolution with internal damage is also described and results are presented. Developments of this strategy are suggested for deriving mechanism-based constitutive laws for engineering applications.
\end{abstract}

\section{INTRODUCTION}

Several barriers in a nuclear waste repository, such as waste immobilisers, backfills and host rocks are porous. While their hydraulic permeability maybe very low at the construction stage, it may evolve over the repository lifetime due to mechanisms that could change the pore space geometry and connectivity. Examples for such mechanisms are cracking, electrochemical and/or bacterial corrosion, and possibly irradiation. Predicting permeability evolution with such changes is critical to the assessment of radionuclide transport. This can be achieved by appropriate pore space models amenable to mechanism-driven changes.

Pore-network models (PNM) offer an advantageous pore space description [1]. PNMs contain a set of pores, e.g. spheres of different radii, connected by throats, e.g. cylinders with different radii. Pores reside at sites of a regular lattice and throats link some neighbouring pores. Early PNMs were based on a cubic lattice with a maximum pore coordination of six [2]. Later experimental studies showed that pores with larger pore coordination numbers (PCN) can be substantial fractions of all coordinated pores [3]. These lead to proposals of PNMs with larger maximum PCN, e.g. 26 in [4], still based on cubic support. Although such PNMs are topologically admissible, they are physically unrealistic because large numbers of throats intersect at points that are not pores. Further, for a given average PCN the existing models reproduce only one PCN spectrum, i.e. fractions of pores coordinated by different numbers of throats. This spectrum may not correspond to experimentally determined spectra, such as in [3].

This work has four objectives. Firstly, to present PNMs based on a novel lattice support with truncated octahedral cells and provide physical justification for its selection. Secondly, to demonstrate the abilities of the models to produce various PCN spectra and to predict experimentally measured permeability. Thirdly, to illustrate how the models can be used to study evolution of permeability with pore space changes. Fourthly, the future applications of the models for deriving constitutive laws will be discussed. 


\section{THEORY AND EXPERIMENT}

We consider the meso-scale of a porous medium to be a length scale dictated by the distances between the pores. At this scale, the throats are fluid conduits and the flow is a continuum phenomenon. The relevant meso-scale properties are the connectivity of pores and the conductivity of throats. We conjecture that these mainly control the emergent (macroscopic) property fluid permeability. We present below a model that captures this philosophy in a topologically suitable and computationally simple manner, together with experimental data used to calibrate the model and demonstrate its potential.

\section{Model and method}

At the meso-scale, the pore space is characterised by a pore set and a throat set. A 3D domain containing a pore set can be tessellated into cells using Voronoi construction, where pores reside at the cells centres. Pores in adjacent cells are linked by members of the throat set. This suggests that a good pore space description can be achieved by analysis of Voronoi diagrams of point sets with random spatial distribution. Kumar et al [5] have performed a large series of simulations and produced a statistical picture of the possible cell topologies. This provides an insight into the selection of a regular shape which can be used as a support for PNM construction. Out of the four possible regular solids filling the space compactly, the truncated octahedron offers the best fit to the average cell topology reported. Figure 1 shows the unit cell and an example of PNM support used in this work. An important feature is the presence of two throat types: $T_{1}$ normal to the cells square boundaries with length $L_{1}$, considered as the model length scale, $L$; and $\mathrm{T}_{2}$ normal to the cells hexagonal boundaries with length $L_{2}=3^{1 / 2} L / 2$.
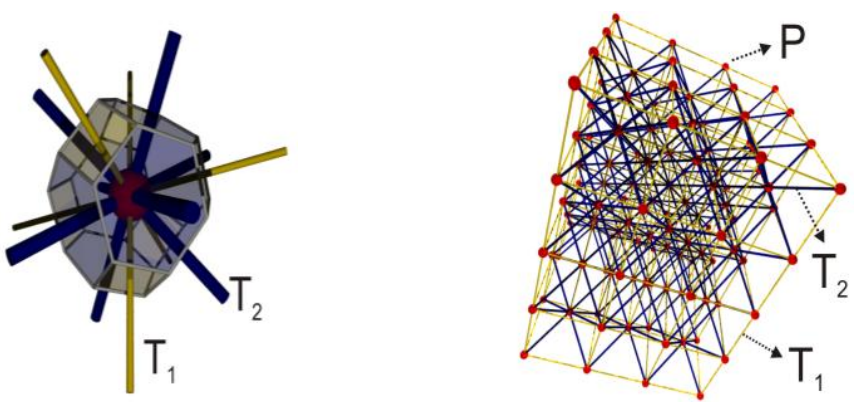

Figure 1. Unit cell with all possible throats (left) and supporting network for pore space construction (right). $\mathrm{P}$ denotes a pore; $\mathrm{T}_{1}$ and $\mathrm{T}_{2}$ denote the two different types of throats.

The construction starts with generation of pore radii, $R_{\alpha}$, according to a pre-defined pore size distribution (PSD). Next, throats are seeded using pre-defined elimination numbers $0<E_{1}<1$ and $0<E_{2}<1$ for the two throat types, respectively. For each possible throat, a random number $0<E<1$ is generated and if $E<E_{i}(i=1,2)$ the throat is accepted as conductive, otherwise rejected as non-conductive. This results in pores being coordinated by various numbers of conductive throats between 0 and 14 and an average PCN, $Z$, for the entire system. A pre-defined $Z$, usually determined experimentally, can be achieved with a range of $E_{1}$ and $E_{2}$ selections. Figure 2 shows an example of two possible PCN spectra giving the same $Z$. This illustrates the important ability of the model to be tuned to known PCN spectrum, which cannot be achieved with existing models based on cubic support. 

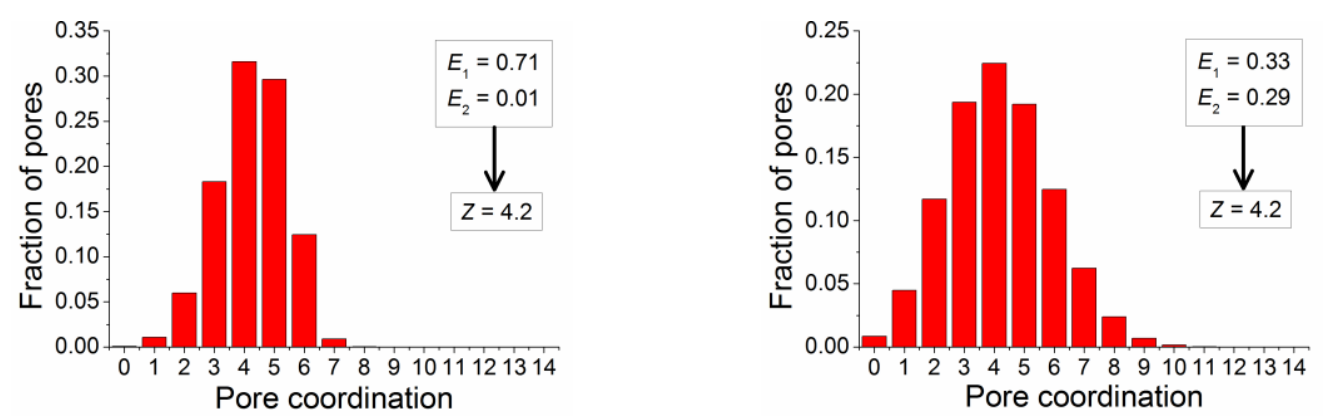

Figure 2. Fractions of pores with different coordination numbers in a network with average pore coordination $Z=4.2$. These are obtained with $E_{1}$ and $E_{2}$ as depicted.

The radii of the conductive throats, $R_{\alpha \beta}$, are generated according to a pre-defined throat size distribution (TSD). The non-conductive throats are also ascribed a very small radius, relative to conductive throats, which is necessary for future studies of pore space evolution. A model with $N_{x}, N_{y}, N_{z}$ sites in the principal lattice directions represents a volume $V=N_{x} \cdot N_{y} \cdot N_{x} \cdot L^{3}$. For a given porosity, $\rho$, the length scale of this model, $L$, is determined from

$\left(\rho N_{x} N_{y} N_{z}\right) L^{3}=(4 \pi / 3) \sum_{\alpha}^{\text {pores }} R_{\alpha}^{3}+\left(\pi \sum_{\alpha, \beta}^{\text {throats }} \lambda_{\alpha \beta} R_{\alpha \beta}^{2}\right) L$

where $\lambda_{\alpha \beta}=1$ for $\mathrm{T}_{1}$, and $\lambda_{\alpha \beta}=3^{1 / 2} / 2$ for $\mathrm{T}_{2}$. For the model thus constructed, we assume laminar fluid flow in cylindrical throats. The total discharge, $Q_{\alpha \beta}$, of a throat of radius $R_{\alpha \beta}$ and length $L_{\alpha \beta}$ connecting pores $\alpha$ and $\beta$, with pressures $p_{\alpha}$ and $p_{\beta}$, respectively, is given by:

$Q_{\alpha \beta}=\left(\pi R_{\alpha \beta}^{4} / 8 \mu\right)\left(p_{\alpha}-p_{\beta}\right) / L_{\alpha \beta}=c_{\alpha \beta}\left(p_{\alpha}-p_{\beta}\right)$,

where $c_{\alpha \beta}$ is the local throat conductivity and $\mu$ is the fluid viscosity. The conservation of mass at pore $\alpha$ is given by $\Sigma c_{\alpha \beta} \cdot\left(p_{\alpha}-p_{\beta}\right)=0$, where the sum is taken over all throats coordinating the pore. Assemblage of all pores gives a linear system of equations $\mathbf{C} \cdot \mathbf{p}=\mathbf{f}$, where $\mathbf{C}$ is the throat conductivity matrix, $\mathbf{p}$ is the vector of pore pressure values, and $\mathbf{f}$ is the external force vector, reflecting boundary conditions. This system describes a steady-state fluid flow through the model [6], which corresponds to the condition for experimental measurement of permeability.

\section{$\underline{\text { Experimental data }}$}

We have selected two different sandstones as model materials: Hollington (HSS) and Tennessee (TSS). The porosity and permeability of these samples were measured using a ResLab digital helium porosimeter and gas permeameter, respectively, using standard-size cylindrical core samples. Results relevant to this work are listed in Table I: porosity for the model construction; steady-state pressures during permeametry for prescribing boundary conditions; and calculated experimental permeability for model calibration.

Thirty micron-thick sections of the sandstones were used to examine the pore space structure with a standard petrographical optical microscope. One hundred digital images from each sandstone thin section, covering areas of $187 \mathrm{~mm}^{2}$, were analysed by image analysis software (ImageJ). Pore space features down to $3 \mu \mathrm{m}$ have been resolved. In HSS 250 pores have been resolved ranging from 5 to $235 \mu \mathrm{m}$ and throats of sizes between $3 \mu \mathrm{m}$ and $30 \mu \mathrm{m}$ have 
been observed. In TSS 630 pores were resolved, ranging from $3 \mu \mathrm{m}$ to $45 \mu \mathrm{m}$ but throats were not observed at this resolution. This suggests that the TSS throats responsible for the measured permeability are much narrower than the minimum pore size. In the range where throats were resolved in HSS, the shape of the TSD has been found to be similar to the shape of the PSD. We therefore assume that, for both samples, the TSD has the same shape as the PSD with maximum throat size being a factor, $\sigma$, times the minimum pore size. The observations suggest that $\sigma>1$ (possibly 5-6) for HSS and $\sigma<1$ for TSS. Pore and throat sizes are distributed in the model using cumulative PSD and uniformly generated random numbers. The cumulative PSD were constructed from the corresponding PSD by standard statistical ranking and are given in Fig. 3.

Table I. Experimental macroscopic properties of sandstones: porosity and permeability

\begin{tabular}{|l|l|l|l|l|}
\hline Sandstone & Porosity $(\boldsymbol{\%})$ & $\begin{array}{l}\text { Gas inlet } \\
\text { pressure }(\mathbf{M P a})\end{array}$ & $\begin{array}{l}\text { Pressure } \\
\text { difference }(\mathbf{M P a})\end{array}$ & $\begin{array}{l}\text { Permeability } \\
\left(\boldsymbol{\mu m}^{\mathbf{2}}\right)\end{array}$ \\
\hline Hollington & 24.7 & 0.2 & 1.03 & $4.6 \times 10^{-3}$ \\
\hline Tennessee & 8.6 & 0.2 & 17.5 & $1.8 \times 10^{-6}$ \\
\hline
\end{tabular}
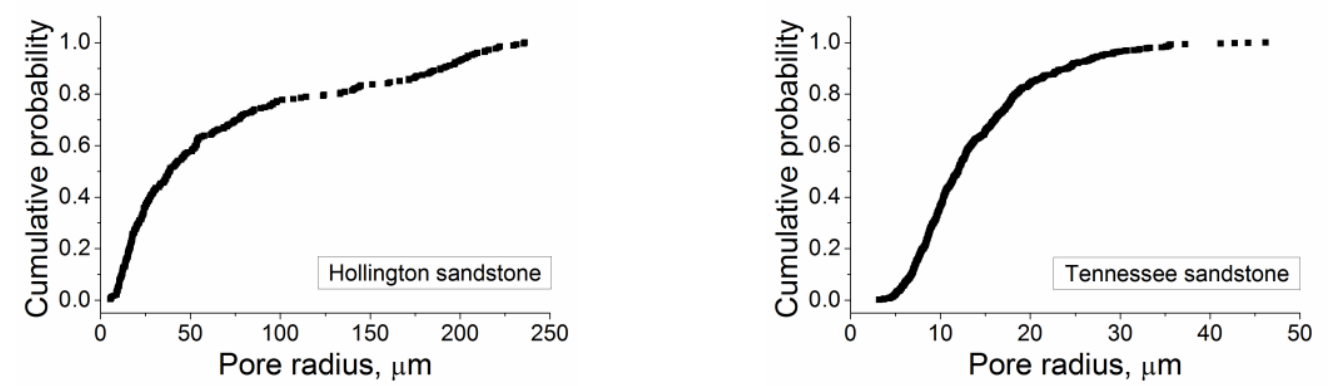

Figure 3. Cumulative pore size distribution for HSS (left graph) and TSS (right graph).

The 2D image analysis is not sufficient to extract reliable pore coordination data. In HSS pores coordinated by one to five throats were observed. The statistics of these, however, cannot be used for determining an average PCN and spectrum for the 3D model. For both sandstones, therefore, we have used a literature value, $Z=4.2$, typical for sandstones [7]. The HSS results hint at selecting a model with a wider PCN spectrum. Thus in the results presented next, the distribution shown in the right plot of Figure 2 has been used.

\section{RESULTS AND DISCUSSION}

The model used occupies a region $\left(0 \leq X_{1} \leq 20 L, 0 \leq X_{2} \leq 10 L, 0 \leq X_{3} \leq 10 L\right)$ with respect to a coordinate system $\left(X_{1}, X_{2}, X_{3}\right)$ oriented along the principal directions of the unit cell. To represent the permeability tests, pressures $p_{\text {in }}$ and $p_{\text {out }}\left(p_{\text {in }}>p_{\text {out }}\right)$ are prescribed at pores on planes $X_{1}=0$ and $X_{1}=20 L$, respectively, and zero fluxes at pores on planes $X_{2}=0, X_{2}=10 L, X_{3}=0$, and $X_{3}=10 L$. The model permeability, $k$, is found from the total inflow $Q_{i n}$ across $X_{1}=0$, or equivalently outflow $Q_{\text {out }}$ across $X_{1}=20 L\left(Q_{\text {out }}=Q_{\text {in }}\right)$ and the Darcy's law:

$k=\mu Q_{\text {in }} /\left(p_{\text {in }}-p_{\text {out }}\right)=\mu Q_{\text {out }} /\left(p_{\text {in }}-p_{\text {out }}\right)$. 


\section{Model calibration}

The ratio between the maximum throat size and the minimum pore size, $\sigma$, has been varied within certain limits to obtain the measured permeability for both sandstones. For each $\sigma$, 30 simulations were performed with random distribution of pores and throats, subjected to the constraints PSD, $E_{1}=0.33$ and $E_{2}=0.29$. Figure 4 shows the results of all simulations together with the mean permeability values given by thick lines. Depicted on the graphs are also the measured permeability values and the calibrated model parameters. The model is clearly capable of reproducing very different systems in terms of porosity and permeability values. It is interesting to note that the calculated model length scales, $L$, are roughly twice the maximum pore sizes of the two sandstones (see Figure 3).
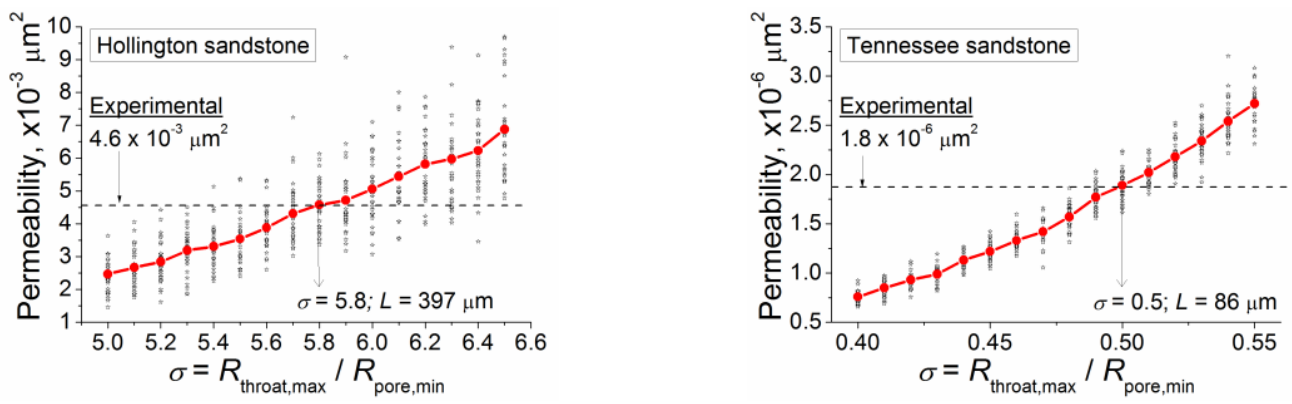

Figure 4. Model calibration for HSS (left graph) and TSS (right graph). Simulation results shown with hollow starts; predicted mean permeability shown with thick line.

\section{Permeability evolution}

The permeability evolution with possible pore space changes is of key interest. We discuss how changes due to various mechanisms can be accounted for in the next sub-section. Here we illustrate the model predictions based on simplifying assumptions. Mimicking mechanical damage, we assume solid phase failure around the pore which is simultaneously largest and best connected at a given simulation step. This pore is not considered for further failure and its coordinating throats are enlarged proportionally, so that their total volume is increased by the pore volume. This leads to changes in porosity and permeability, recalculated after each failure event. Figure 5 shows the results of simulations performed with this strategy. The graphs suggest that increase of permeability with increase of porosity is largely independent of the initial values (sandstone type) and probably dictated by connectivity $\left(E_{1}, E_{2}\right)$ alone. The deviations for the two sandstones can be attributed to differences in the two PSD (see Figure 3 ).
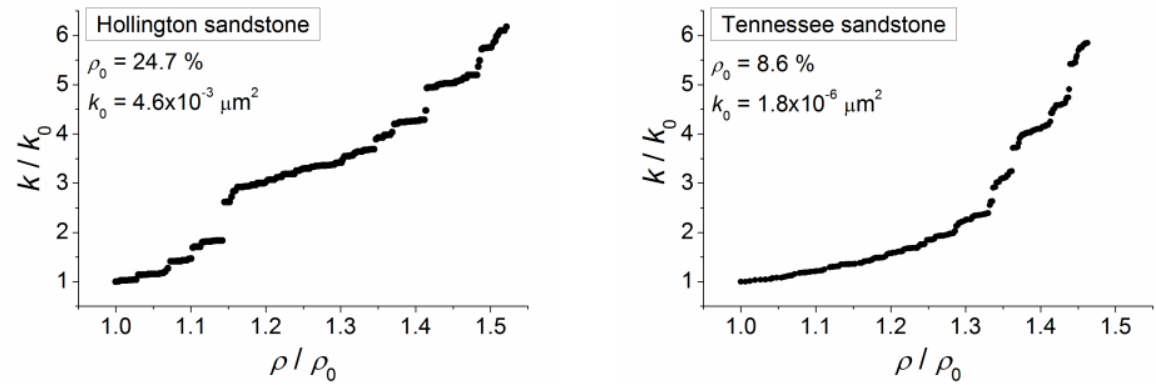

Figure 5. Evolution of permeability with damage for HSS (left graph) and TSS (right graph). 


\section{Model development}

The model allows for additional features: (i) connectivity anisotropy and/or heterogeneity via assignment of different elimination numbers to throats in different directions and/or domains; (ii) conductivity anisotropy and/or heterogeneity via assignment of different conductivity values to throats in different directions and/or domains; (iii) material texture via assignment of different cell sizes in the principal directions. Once a model, reflecting a pore space is constructed and calibrated, pore space changes due to various mechanisms can be introduced with dual models.

For mechanisms operating in the pore space, e.g. corrosion, the dual model geometry coincides with the pore space. The dual model describes a mechanism as a continuum process with its own boundary conditions but can be affected by and affect the primary model, i.e. account for and cause changes in the pore space. For mechanisms operating in the solid phase, e.g. nucleation and growth of micro-cracks, the dual model geometry differs from the pore space. It considers solid phase grains as domains free of pores, while pores reside at grain boundaries. In the dual model grain centres are linked to neighbours by bonds "containing" pores of the primary model. A bond's strength is dictated by its pore size. The dual model can be subjected to various deformations due to external loads or internal pressures from the pore space. Damage can be simulated by bonds elimination according to a failure criterion and communicated to the primary model. This strategy will produce a mechanism-based, rather than phenomenological, constitutive law for permeability evolution for continuum/engineering applications. Use of several models for competing mechanisms provide an opportunity for multi-physics studies.

\section{CONCLUSIONS}

The objectives of this work have been achieved. We have: (i) presented and justified a model of pore space that is topologically accurate and computationally simple for predicting permeability; (ii) demonstrated that the model can be calibrated for permeability predictions of two very dissimilar pore systems with a minimal amount of experimental input; (iii) illustrated the use of the model for permeability evolution with pore space changes (or solid phase damage); and (iv) discussed prospective developments for deriving continuum constitutive laws.

\section{REFERENCES}

1. M.J. Blunt, M.D. Jackson, M. Piri and P.H. Valvatne, Adv. Water Resour. 25, 1069 (2002).

2. P.C. Reeves and M.A. Celia, Water Resour. Res. 32, 2345 (1996).

3. A.S. Al-Kharusi and M.J. Blunt, J. Petrol. Sci. Eng. 56, 219 (2007).

4. A. Raoof and S.M. Hassanizadeh, Transp. Porous Media 81, 391 (2010).

5. S. Kumar, S.K. Kurtz, J.R. Banavar, and M.G. Sharma, J. Stat. Phys. 67, 523 (1992).

6. P. Van Marcke, B. Verleye, J. Carmeliet, D. Roose and R. Swennen, Transp. Porous Media 85, 451 (2010).

7. W.B. Lindquist, A. Venkatarangan, J. Dunsmuir and T.-F. Wong, J. Geophys. Res. 105, 21509 (2000). 